\title{
Development of a new assessment tool for cervical myelopathy using hand tracking sensor - Part 1: validity and reliability
}

\section{Authors:}

\section{Abdulhadi Alagha}

Academic Orthopaedics, Trauma and Sports Medicine, The University of Nottingham, Queen's Medical Centre, Nottingham NG7 2UH, UK

\section{Mahmoud A Alagha}

Medical, Veterinary and Life Sciences College, University of Glasgow

Dental Hospital, Glasgow G2 3JZ, UK

\section{Eleanor Dunstan}

Centre for Spinal Studies and Surgery, Queen's Medical Centre, Nottingham NG7 2UH, UK

\section{Olaf Sperwer}

VRmed Ltd., Rosewood House, Radcliffe on Trent, Nottingham NG12 2BQ, UK

\section{Kate A Timmins}

School of Sport \& Exercise Science, University of Lincoln, Lincoln LN6 7TS, UK

\section{Bronek M Boszczyk (Correspondence)}

Centre for Spinal Studies and Surgery, Queen's Medical Centre, Nottingham NG7 2UH, UK

E-mail: bronek.boszczyk@nuh.nhs.uk

Ethical Approval: Obtained by The University of Nottingham.

Ethics Reference No: F12032015 SoM Spinal Surg

Funding: The study was funded by Nottingham University Hospitals (Spine Research). Bbraun and VRmed supported the design of the software. The companies had no role in the design, interpretation or reporting of the study. The authors declared no conflict of interest. 


\begin{abstract}
Purpose To assess the reliability and validity of a hand motion sensor, Leap Motion Controller (LMC), in the 15-second hand grip-and-release test, as compared against human inspection of an external digital camera recording.

Methods Fifty healthy participants were asked to fully grip-and-release their dominant hand as rapidly as possible for two trials with a 10-minute rest in-between, while wearing a non-metal wrist splint. Each test lasted for 15 seconds, and a digital camera was used to film the anterolateral side of the hand on the first test. Three assessors counted the frequency of grip-and-release (G-R) cycles independently and in a blinded fashion. The average mean of the three was compared with that measured by LMC using the Bland-Altman method. Test-retest reliability was examined by comparing the two 15 -second tests.
\end{abstract}

Results The mean number of G-R cycles recorded was: $47.8 \pm 6.4$ (test 1, video observer); $47.7 \pm 6.5$ (test 1, LMC); and 50.2 \pm 6.5 (test 2, LMC). Bland Altman indicated good agreement, with a low bias ( 0.15 cycles) and narrow limits of agreement. The ICC showed high inter-rater agreement and the coefficient of repeatability for the number of cycles was \pm 5.393 , with a mean bias of 3.63 .

Conclusions LMC appears to be valid and reliable in the 15-second grip-and-release test. This serves as a first step towards the development of an objective myelopathy assessment device and platform for the assessment of neuromotor hand function in general. Further assessment in a clinical setting and to gauge healthy benchmark values is warranted.

Key Words: Cervical Myelopathy, Virtual Reality, Leap Motion, Grip and release test, Reliability, Validity 


\section{Introduction}

Cervical myelopathy represents a compression of the spinal cord at the level of cervical vertebrae leading to neurological loss [9]. Cervical myelopathy patients' symptoms are thought to deteriorate in a stepwise pattern [6], therefore prompt recognition of any early decline in function is crucial for optimal treatment.

There have been a number of tools proposed to diagnose cervical myelopathy and to quantify its severity. Several symptom-based grading scales exist (such as Nurick [21], Ranawat [24], Cooper and Epstein [7], Harsh [12], the European Myelopathy score (EMS) [13] and the Japanese Orthopaedic Association (JOA) Score [30]), but these have been criticised for lacking sensitivity [14]. Acknowledging the limitations of subjective symptom-based scales, several authors have suggested quantitative tests. These include: the 30-minute walking test devised by Singh and Crockard [25]; the Ten-second step test [20] (an improvement on the time-consuming 30-minute test); or the Apposition of the thumb test [17]. These tests, although promising as quantitative tools, were found to be of limited use in advanced-stage patients, where walking or the initial stages of the test are not viable.

The literature shows that digital clumsiness precedes any other weakness in cervical myelopathic patients, thus manual dexterity evaluation is potentially the most favourable means of quantitative assessment [22]. Ono and colleagues [22], observing the neurological deterioration of motor and sensory functions of the hand in cervical myelopathy ('the myelopathy hand'), developed a 10-second grip and release test, based on counting the number of flexion-extension cycles in 10 seconds. Hosono et al [14] extended this to 15 seconds in order to perform a validation of the method against JOA scores [15]. This 15-second grip-and-release test was demonstrated to be both reliable and valid. However, the method required recording of hand movements using a digital camera for later examination (to count the cycles). This reduces its practicality in clinical settings.

The Leap Motion Controller (LMC) is a small, remote-sensing (touch-free), Virtual Reality (VR) computerstimulated hand-tracking USB technology system, developed by an American company in 2013. It has been found to be an accurate and a robust sensor [29]. It offers possibilities of quantitatively and automatically measuring the motor function of the hand. The current study serves as a first step toward the development of this novel objective tool for assessing the severity of cervical myelopathy.

\section{Materials and methods}

The study design was a proof of concept clinical trial aiming to incorporate LMC with the 15-second grip and release test. The hypothesis was that the LMC would be both valid and reliable for this purpose.

Fifty participants were recruited from a student population via advertisements and class announcements. Fifty was identified as the minimum sample size for the desired precision of the estimates in the Bland-Altman method [2]. Ethical approval was obtained by The University of Nottingham Ethics committee.

Participants gave informed consent, confirmed their compliance with the eligibility criteria (Table 1), and filled out a demographic information sheet. An inconvenience allowance was provided, and a unique code was allocated for each participant, to allow blinded analyses. The study was funded by Nottingham University Hospitals (Spine Research). Bbraun and VRmed supported the design of the software. The companies had no role in the design, interpretation or reporting of the study. The authors declared no conflict of interest. 
Table 1 Inclusion and Exclusion Criteria

\section{Inclusion Criteria}

- Healthy students

- Males and Females

- Age 18-35 years

- Able to give informed consent

\section{Exclusion Criteria}

- Individuals suffering from locomotor or neurological disorders such as rheumatoid arthritis (RA), cerebral palsy (CP), cervical or thoracic myelopathy, motor neuron disease, degenerative disease (osteoarthritis) or neuromuscular disease

- Individuals with congenital or syndromic hand or spine deformities

- Individuals who have sustained displaced hand or cervical fractures

- Individuals with previous history of cervical spine or hand surgery

- Individuals with sensory or motor symptoms in the spine or hand region (numbness, clumsiness, motor weakness)

- Individuals with special communication needs

Wrist Splinting

Wrist splints were used to prevent paradoxical wrist trick motion [15, 23]: specifically excessive wrist extension during the grip (finger flexion) phase. PROMEDICS® non-metal braces (small, medium, large, $x-$ large) were used (Fig1), because metals hamper the performance of Virtual Reality devices [8]. Sizing the splint for each participant followed the Storm Wrist Brace Sizing Guide, which was based on forearm and hand circumference measurements.

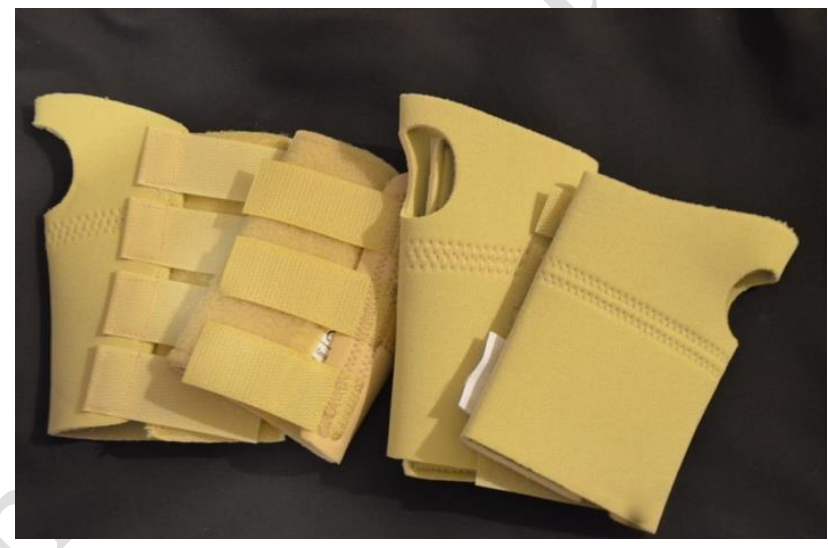

Fig1 PROMEDICS® non-metal wrist splints

Apparatus and Software

A laptop computer (MSI GT72 Dominator Pro-Dragon Edition, MSI-NL., Eindhoven, China) was used to display a visual VR programme (VR-MED), developed by VRmed Ltd., Nottingham, UK. VRmed Ltd. used the game engine software Unity3D (Unity Technologies San Francisco, USA) and Phyton code to develop the interactive vr test application for the LMC, displaying and measuring the positions of fingers at each 0.02 second. A Leap Motion Controller (Version 3.3.1 Orion, Leap Motion Inc., San Francisco, CA, US) was connected to the laptop and firmly attached to a table, 2.95 inches $(7.5 \mathrm{~cm})$ in front of the keyboard, centered to the screen, and 3.93 inches $(10 \mathrm{~cm})$ from the edge of the table. 
A chair was placed 20 inches $(50 \mathrm{~cm})$ from the edge of the table at an appropriate height $(\mathbf{F i g} 2)$. The dominant arm of the participant was placed in a pronated position with the elbow flexed to 90.

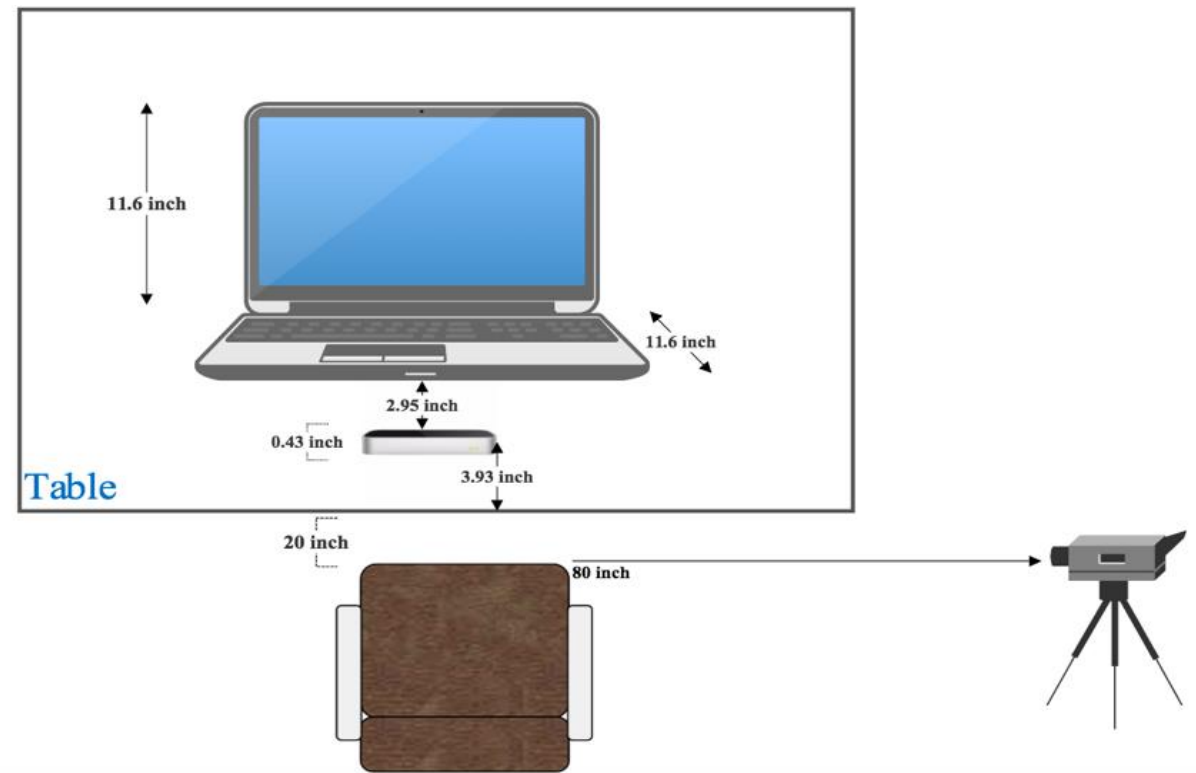

Fig2 Apparatus of study: A chair in front of a table and 80 inches away from the digital camera

Eighty inches (2 m) from the controller, a digital camera (D5100, Nikon, Tokyo, Japan) on a tripod, Manfrotto Befree, was set in a "movie" mode to capture the ulnar dorsal aspect of the hand and wrist. The forearm and hand only were included in the camera's field of view to blind observers [15]. The positions of all adaptable components of the apparatus remained fixed for the duration of the experiment. Lighting and temperature (2224 degrees Celsius) in the room were kept constant [10]. The coordinate system and location of the device were set according to the leap motion user guidelines [19], where the Z-axis pointed towards the participant, the Xaxis to his/her right side and the Y-axis facing upwards (Fig3).

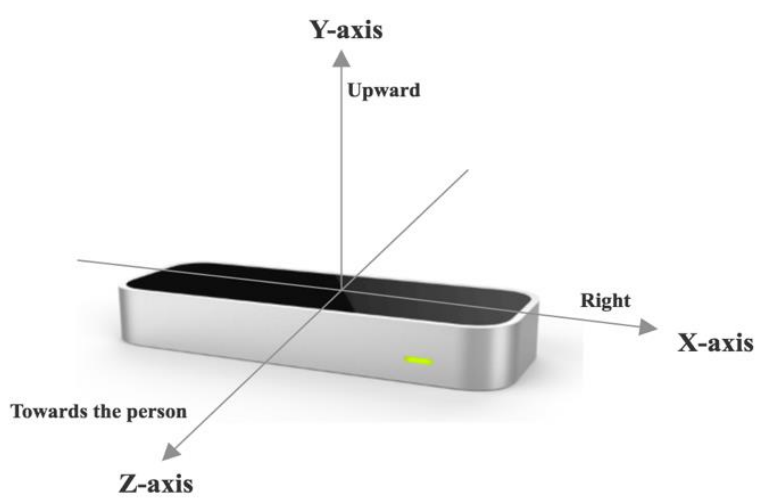

Fig3 Leap Motion Controller Axes

The hand was positioned 3.93 inches $(10 \mathrm{~cm})$ from the controller's side, rather than above it, as it was shown to have better accuracy in a previous study [18], and 7.87 inches $(20 \mathrm{~cm})$ above the controller [19]. The LMC software, VR-MED, analysed the motion of the hand rather than the number of cycles: it captured five positions, where each one correlated to 20 degrees of motion, every 0.02 seconds. A full flexion half-cycle was the motion from position 1 to position 5 (Online Resource 1), while the motion from position 5 to position 1 was a full extension half-cycle (Online Resource 2). Therefore, a full cycle included 8 motions and a full half cycle included 4 motions. The duration of a test could be chosen before starting and the test showed a schematic hand throughout its duration (Fig4). 


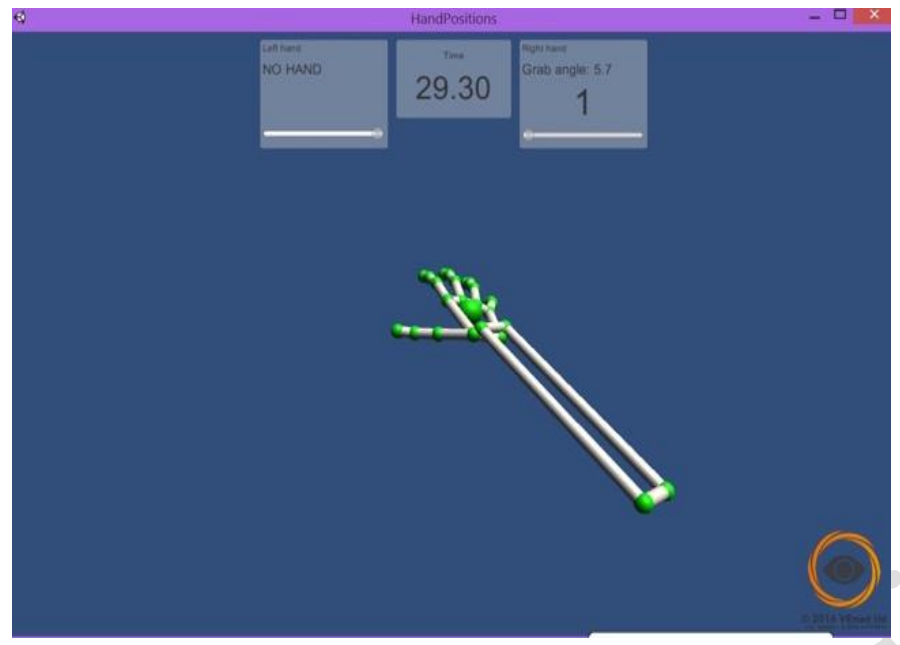

Fig4 Screen display of the VR-Med test

\section{Assessing the outcomes:}

Before starting the measurement, participants received a short tutorial about performing a full cycle grip and release. Following this, participants were asked, with their splinted dominant hand pronated, to fully grip and release (G-R) their fingers as rapidly as possible for the duration of each test. Participants performed two tests, with a 10-minute rest in-between:

1. 15-second test to assess validity

2. 15 -second test to assess reliability.

The digital camera captured the first 15 -second test. Recordings were slowed to $0.25 \mathrm{x}$ on playback and analysed by three independent assessors, using VLC media player, Version 2.2.1-Terry Pratchett (Weatherwax). Observers counted the total number of cycles over $15 \mathrm{~s}$ and the average number of each participant across the $15 \mathrm{~s}$ was computed. The LMC software created a spreadsheet for each undertaken test showing the hand position for each 0.02 s interval. G-R total number of cycles over $15 \mathrm{~s}$ and the number of motions in each cycle (magnitude of motion) were manually derived from this in the two tests.

\section{Statistical Analyses:}

The interrater reliability of the three video observers was analysed by a two-way mixed Intraclass Correlation Coefficient (ICC). The validity of the LMC in capturing the number of grip and release cycles was assessed using the Bland-Altman method, with video observations of the first 15 -second test taken as the reference measurement. The mean frequency of the three video assessors was taken as the reference. Test-retest reliability, for the frequency of cycles as well as the motions' extent, was assessed with the coefficient of repeatability (CR) [4]. Descriptive statistics are also presented. Cycle count data, but not magnitude of motion data, were normally distributed.

IBM SPSS Statistics for Macintosh, version 22.0 was used to analyse all data [16].

\section{Results}

Fifty participants [Males: 27 (54\%), Females: 23 (46\%)], of various ethnicities, met the eligibility criteria and agreed to participate in the study. Participants' ages ranged from 19 to 34 years (Mean=24.24, SD=3.28). 46 participants were right-handed $(92 \%)$ and four left-handed $(8 \%)$. The mean number of G-R cycles recorded was: $47.8 \pm 6.4$ (test 1 , video observer); $47.7 \pm 6.5$ (test $1, \mathrm{LMC}$ ); and 50.2 \pm 6.5 (test 2, LMC). The median magnitude of motion was: 7.46 (IQR 6.70-7.89) (test 1, LMC); and 7.65 (IQR 7.17-7.91) (test 2, LMC).

\section{Inter-rater reliability}

The ICC showing agreement between the three video observers was high: $\mathrm{ICC}=0.998$ (95\%CI=0.997-0.999), $\mathrm{p}<0.001$.

Validity

Fig5 shows a scatter plot of the number of cycles counted using the LMC versus the number of cycles counted from the video recording. 


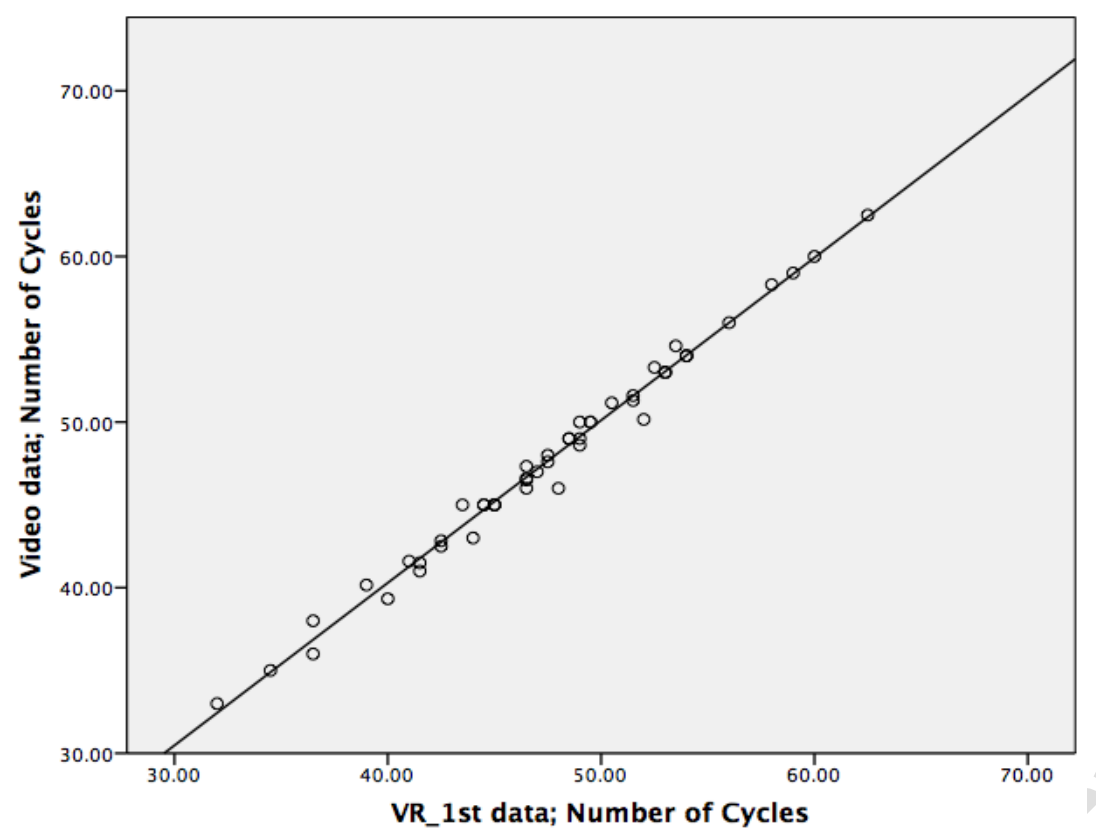

Fig5 Relationship between Leap Motion Controller and human inspection using a digital camera); $r=0.995$

The Bland-Altman plot (Fig6) showed a bias of 0.15 cycles $(95 \% \mathrm{CI}=0.10$ to 0.20$)$. The upper and lower limits of agreement were -1.16 and 1.46 cycles respectively.

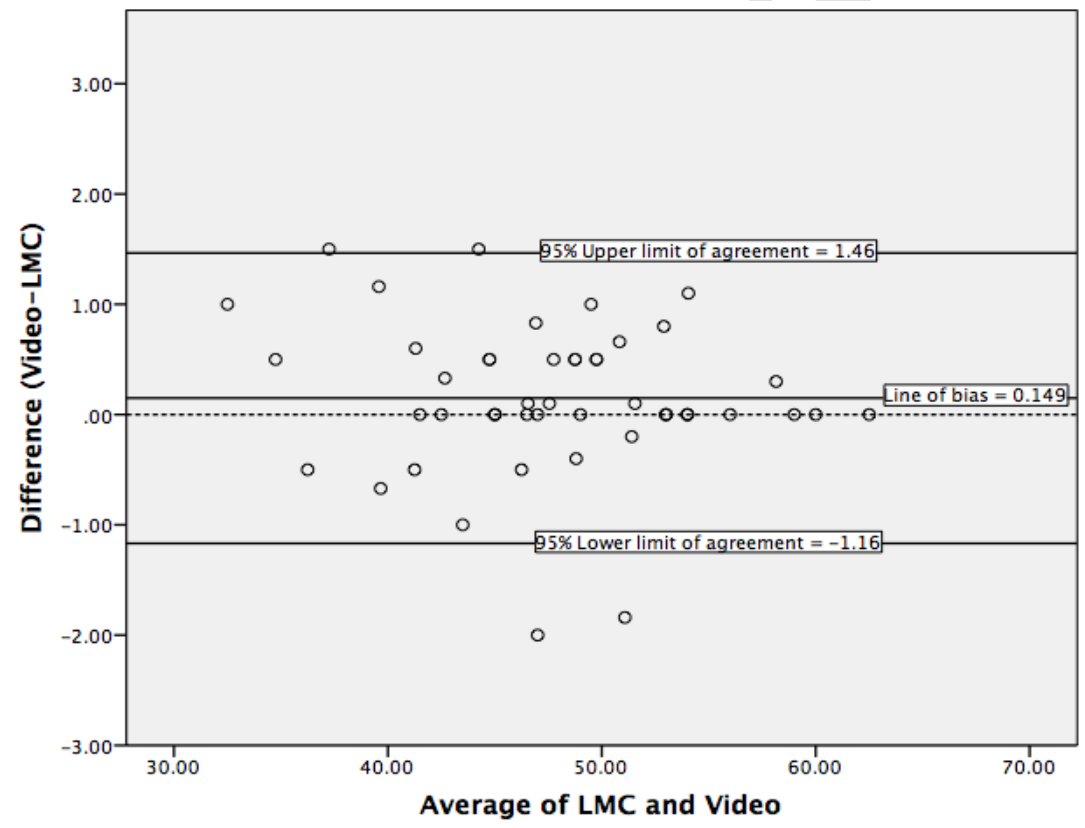

Fig6 Limits of agreement for the number of grip-and-release cycles between leap motion controller (LMC) and visual inspection of video files (Video)

Test-retest reliability

Fig7 shows the number of cycles counted by the LMC in the first 15-second test compared to the second 15second test. The $\mathrm{CR}_{\text {Cycles }}$ was \pm 5.393 , with a mean bias of 3.63 . 


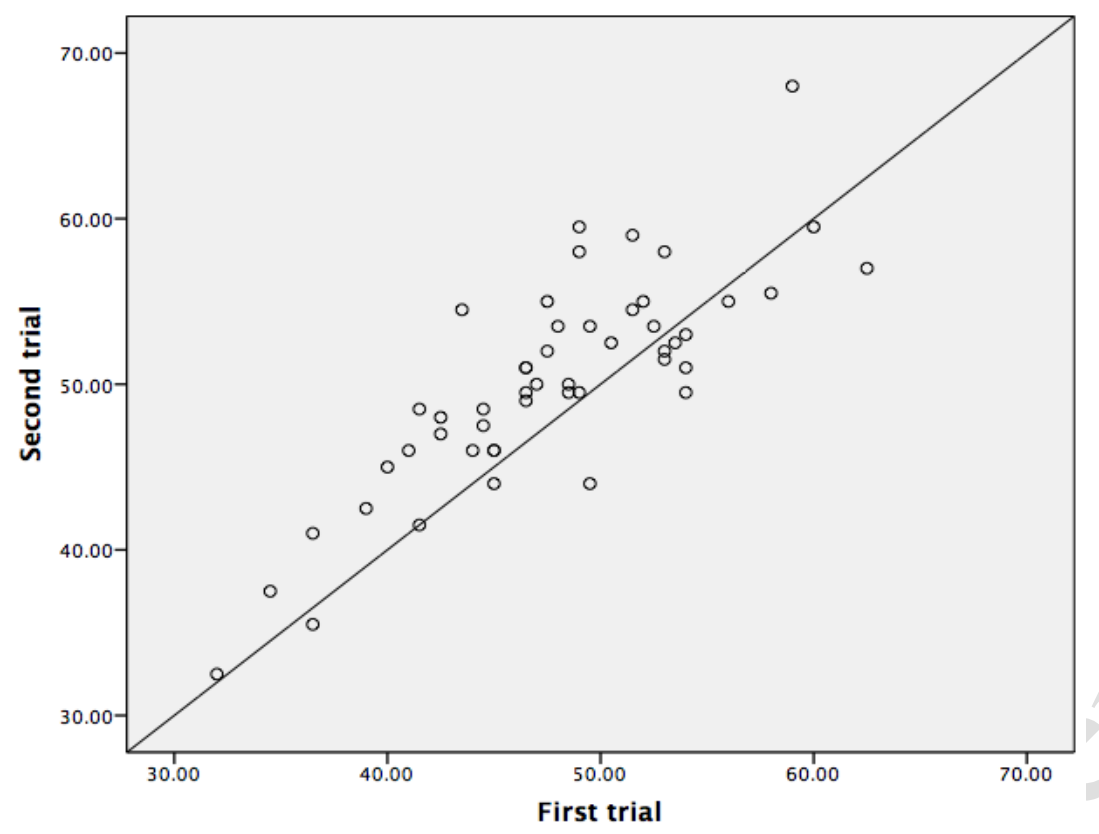

Fig7 Relationship between the $1^{\text {st }}$ trial and the $2^{\text {nd }}$ trial for the number of cycles; a scatterplot showing line of equality

\section{Discussion}

This study aimed to assess the validity and reliability of the LMC for use in a 15-second grip and release test in healthy participants. The findings suggest that the leap motion controller (LMC) and its software are reliable and valid in the assessment of the 15-second hand grip and release test.

Compared to the video observations, the LMC showed good agreement. The results imply the LMC tends to slightly underestimate the number of G-R cycles in a 15-second test, with an observed bias of 0.15 cycle. However, this represents just $0.31 \%$ measurement error for an average number of cycles of 47.7. Furthermore, the even spread of data points across the Bland Altman plot suggests no systematic difference across the range of cycle counts. The limits of agreement ( -1.16 and 1.46 cycles) were also relatively narrow, and indicate that for approximately $95 \%$ of cases, the number of G-R cycles measured by the LMC will differ from video inspection by between -1.16 and 1.46 cycles.

The high ICC for inter-rater reliability is in agreement with previous research [14] and indicates that video observation is an appropriate reference measurement for this validation. It is also worth noting that the 15second grip-and-release test via recorded video has been previously validated against the Japanese Orthopaedic Association (JOA) score [14, 27].

There appeared to be greater variability in the repeated G-R test measurements using the LMC than between the comparison of the LMC against video observation. The coefficient of repeatability (CR) was \pm 5.4 . An observed difference should be at least as large as the CR to be considered real. Therefore, G-R test scores would need to differ by more than five and half cycles to be considered to reflect a real difference. This has potential implications for the use of LMC in gauging the progression of cervical myelopathy. However, it is possible that this CR reflects fatigue or other carry-over effects from the study design. The literature lacked evidence about the most appropriate time interval between the 15-second G-R test administrations. A 10minute rest was chosen as being pragmatic in this study. Consequently, if the rest between tests was insufficiently long, this could have increased the coefficient of repeatability and its limits of agreement [1]. It may be worthwhile to introduce a longer period of rest, such as 24 hours, between repeated tests in a future 
study to establish the potential effect of fatigue. Test-retest reliability has previously been found to vary in repeated administrations of the 10-second G-R test: Wada et al [28] reported the intra-observer reliability of the 10 -second G-R test in healthy subjects via human inspection to be $r=0.78$ (Spearman's, $n=70$ ).

There is no statistical approach to decide whether the CR is large or small [26]. Mean bias and CR should always be interpreted according to their clinical relevance [5]; this will assist physicians to judge whether the observed change in patient's score is true [3]. The Minimum Clinically Important Difference (MCID) needs to be established on clinical grounds instead of statistical analysis [11]. Currently, there is no consensus regarding the MCID of the 15-second G-R test. Ono and colleagues [22] stated that less than twenty G-R cycles per 10 seconds should be regarded as symptomatic myelopathy. In a different study, a threshold of 21 or 22 cycles was reported between cervical myelopathy patients and healthy individuals for the 10-second G-R test [28]. Yukawa et al. [31] estimated the value (Average- $2 * \mathrm{SD}$ ) to represent an index of a pathological state in the 10second G-R test for each age group in the Japanese population. An average number of less than twenty cycles in normal subjects above sixty years old was reported. This opposes Ono et al's suggestion [22], and highlights the importance of taking into account variation in the number of G-R cycles associated with ageing. Further research into the use of the LMC for the 15-second G-R test in a wider range of age groups is needed - in particular the age range of 40 to 70 years, the typical age range of myelopathy patients. Studies amongst cervical myelopathy patients themselves are also necessary.

Nevertheless, the 15 -second G-R test has been validated against the JOA score $[14,27]$. If the LMC is able to count the number of cycles as accurately as video observation, this implies it is at least as good as video observation for clinical use. The leap motion device is relatively low-cost and has the potential to give objective measurements in fifteen seconds, in contrast to recording the test by camera for future playback and analysis. Further research is needed to assess its reliability in a clinical setting, particularly where variables such as light and temperature are likely to vary. This could also address whether or not the aid of a jig or template is necessary to aid positioning in the clinical setting. In addition, the result for each patient could be compared to both standardised benchmark values as well as their own previous performances to allow an objective assessment of a patient's deterioration or improvement.

Lastly, it is vital to note here that the validity of LMC concerning motions' extent was not formally studied in this study, an assumption of its legitimacy was adopted. Our study assumed that LMC is valid regarding the magnitude of motion; however, future studies should investigate such validity embracing an evidence-based approach.

\section{Conclusion}

Leap motion controller and the VR-MED software appear to be valid and reliable in the 15-second G-R test and show great potential as an objective diagnostic tool in clinical settings for cervical myelopathy. To further proof this concept, setting benchmark figures for the number of G-R cycles and magnitude of motion in healthy individuals is warranted. Future studies should investigate the reliability of the LMC in assessing cervical myelopathic patients, followed by prospective clinical trials to examine the neurological improvements after decompression surgery. 


\section{References:}

1. Allen MJ, Yen WM (1979) Introduction to measurement theory. Brooks/Cole, Monterey (CA).

2. Altman D (1991) Practical statistics for medical research. Chapman and Hall, London

3. Beckerman H, Roebroeck ME, Lankhorst GJ, Becher JG, Bezemer PD, Verbeek ALM (2001) Smallest real difference: A link between reproducibility and responsiveness. Qual Life Res 10:571-578. doi: 10.1023/A:1013138911638

4. Bland JM, Altman DG (1986) Statistical methods for assessing agreement between two clinical methods of clinical measurement. The Lancet, 327(8476):307-310

5. Bland JM (2000) An introduction to medical statistics, 3rd edn. Oxford University Press, Oxford

6. Clarke E, Robinson PK (1956) Cervical myelopathy: a complication of cervical spondylosis. Brain, 79:483-510

7. Cooper PR, Epstein F (1985) Radical resection of intramedullary spine and cord tumors in adults: recent experience in 29 patients. J Neurosurg 63:492-499. doi: 10.3171/jns.1985.63.4.0492

8. Craig A, Sherman W, Will J (2009) Developing Virtual Reality Applications: Foundations of Effective Design. Morgan Kaufmann, Massachusetts

9. Durrant D, True J (2002) Myelopathy, radiculopathy, and peripheral entrapment syndromes. CRC Press, Boca Raton, Florida

10. Guna J, Jakus G, Pogacnik M, Tomazic S, Sodnik J (2014) An Analysis of the Precision and Reliability of the Leap Motion Sensor and Its Suitability for Static and Dynamic Tracking. Sensors 14:3702-3720. doi: 10.3390/s140203702

11. Guyatt G, Walter S, Norman G (1987) Measuring change over time: Assessing the usefulness of evaluative instruments. J Chronic Dis 40:171-178. doi: 10.1016/0021-9681(87)90069-5

12. Harsh GR, Sypert GW, Weinstein PR, Ross DA, Wilson CB (1987) Cervical spine stenosis secondary to ossification of the posterior longitudinal ligament. J Neurosurg 67:349-357. doi: 10.3171/jns.1987.67.3.0349

13. Herdmann J, Linzbach M, Krzan M, Dvorak J, Bock W (1994) The European Myelopathy Score. Advances in Neurosurgery 22:266-268. doi: 10.1007/978-3-642-78801-7_43

14. Hosono N, Sakaura H, Mukai Y, Kaito T, Makino T, Yoshikawa H (2008) A simple performance test for quantifying the severity of cervical myelopathy. Journal of Bone and Joint Surgery 90-B(9):1210-1213. doi: 10.1302/0301-620X.90B9.20459

15. Hosono N, Makino T, Sakaura H, Mukai Y, Fuji T, Yoshikawa H (2010) Myelopathy Hand: New Evidence of the Classical Sign. Spine 35(8):E273-E277. doi: 10.1097/BRS.0b013e3181c6afeb

16. IBM Corp (2013) IBM SPSS Statistics for Macintosh, Version 22.0. Armonk, New York.

17. Kapandji A (1986) Clinical test of apposition and counter-apposition of the thumb. Ann Chir Main 5(1):67-73

18. Katahira R, Soga M (2015) Development and Evaluation of a System for AR Enabling Realistic Display of Gripping Motions Using Leap Motion Controller. Procedia Computer Science 60:1595-1603. doi: 10.1016/j.procs.2015.08.269

19. Leapmotion.com (2016) Coordinate Systems. [online] Available at: https://developer.leapmotion.com/documentation/cpp/devguide/Leap_Coordinate_Mapping.html [Accessed 21 August 2016]

20. Nakashima H, Yukawa Y, Ito K, Machino M, Kanbara S, Morita D, Imagama S, Hamajima N, Ishiguro N, Kato F (2011) Validity of the 10-s step test: prospective study comparing it with the 10-s grip and release test and the 30-m walking test. European Spine Journal 20(8):1318-1322. doi: 10.1007/s00586011-1733-6

21. Nurick S (1972) The pathogenesis of the spinal cord disorder associated with cervical spondylosis. Brain 95:87-100. doi: 10.1093/brain/95.1.87

22. Ono K, Ebara S, Fuji T, Yonenobu K, Fujiwara K, Yamashita K (1987) Myelopathy hand. New clinical signs of cervical cord damage. J Bone Joint Surg (Br) 69(2):215-219

23. Ono K (1998) The surgeon's View on Differential Diagnosis in Patients with Cervical Spine Disorders. Cervical Spondylosis and Similar Disorders:297-330. doi: 10.1142/9789812812704_0013

24. Ranawat CS, O’Leary P, Pellicci P, Tsairis P, Marchisello P, Dorr L (1979) Cervical spine fusion in rheumatoid arthritis. J Bone Joint Surg Am 61:1003-1010

25. Singh H, Crockard HA (1999) Quantitative assessment of cervical spondylotic myelopathy by a simple walking test. Lancet 354:370-373. doi: 10.1016/S0140-6736(98)10199-X

26. Vaz S, Falkmer T, Passmore A, Parsons R, Andreou P (2013) The Case for Using the Repeatability Coefficient When Calculating Test-Retest Reliability. PLoS ONE, 8(9):1-7. doi:

10.1371/journal.pone.0073990 
27. Wada E, Yonenobu K, Fukui M, Hirota Y, Kikuchi S, Toyama Y, Kawakami M, Atsushi S (2003) Grip and Release Test (10 Seconds Test) in Healthy Volunteers and Cervical Myelopathy Patients-A Simple Bedside Test for the Assessment of Cervical Myelopathy. Cervical Spine Research Society:112-113. Available at: http://www.csrs.org/grip-and-release-test-10-seconds-test-in-healthy-volunteers-andcervical-myelopathy-patients-a-simple-bedside-test-for-the-assessment-of-cervical-myelopathy/ [Accessed 21 Aug. 2016]

28. Wada E, Yonenobu K, Fukui M, Hirota Y, Kikuchi S, Toyama Y, Kawakami M, Seichi A (2004) Intraobserver Reliability of Grip and Release Test (10-Second Test) in Healthy Volunteers and Cervical Myelopathy Patients. Cervical Spine Research Society:261-263. Available at: http://www.csrs.org/intraobserver-reliability-of-grip-and-release-test-10-second-test-in-healthyvolunteers-and-cervical-myelopathy-patients/ [Accessed 21 Aug. 2016]

29. Weichert F, Bachmann D, Rudak B, Fisseler D (2013) Analysis of the Accuracy and Robustness of the Leap Motion Controller. Sensors 13(5):6380-6393. doi: 10.3390/s130506380

30. Yamauchi H, Hirabayashi K (1994) Scoring system for cervical myelopathy (Japanese Orthopaedic Association). J Jpn Orthop Assoc 68:490-503

31. Yukawa Y, Nakashima H, Ito K, Machino M, Kanbara S, Kato F (2013) Quantifiable tests for cervical myelopathy; 10-s grip and release test and 10-s step test: standard values and aging variation from 1230 healthy volunteers. Journal of Orthopaedic Science 18(4):509-513. doi: 10.1007/s00776-013-0381-6 